\title{
Einführung in das Reisekostenrecht
}

$\mathrm{Zu}$ den Reisekosten gehören grundsätzlich sämtliche Kosten, die in Zusammenhang mit der Reisetätigkeit z. B. eines Arbeitnehmers anfallen. Hierbei ist es unerheblich, ob es sich um inländische oder ausländische Kosten handelt. Sämtliche Kosten müssen per Beleg bei der Reisekostenabrechnung nachgewiesen werden. Eine bloße Aufzeichnung z. B. von Bewirtungskosten reicht nicht aus und verstößt gleichfalls gegen die Vorgaben der GoBD [1].

\section{Exkurs „GoBD“}

Bei der GoBD [1] handelt es sich um ein Schreiben des Bundesfinanzministeriums vom 14.11.2014, welches sich mit der ordnungsgemäßen Erstellung, Erfassung, Archivierung und Auswertung buchhalterischer Vorgänge u. a. mit Hilfe von elektronischen Datenverarbeitungssystemen beschäftigt. GoBD steht für „Grundsätze zur ordnungsmäßigen Führung und Aufbewahrung von Büchern, Aufzeichnungen und Unterlagen in elektronischer Form sowie zum Datenzugriff“"

Zu den Reisekosten zählen gemäß R 9.4 LStR 2015 (Stand 16.03.2019)

- Fahrtkosten

- Verpflegungsmehraufwand

- Übernachtungskosten und

- Reisenebenkosten

Voraussetzung zur Berücksichtigung der Reisekosten ist, dass diese durch eine berufliche Auswärtstätigkeit verursacht wurden. Der Arbeitnehmer ist verpflichtet, die berufliche Auswärtstätigkeit, die Dauer der Reise und den Reiseweg korrekt aufzuzeichnen und die Kosten anhand von geeigneten Unterlagen (z. B. Tankquittungen, Fahrtenbuch) nachzuweisen bzw. glaubhaft zu machen (vgl. R 9.4 LStR 2015, Stand 16.03.2019). 